\title{
Molecular mechanisms of diabetic cardiomyopathy
}

\author{
Heiko Bugger • E. Dale Abel
}

Received: 22 October 2013 / Accepted: 27 December 2013 / Published online: 30 January 2014

(C) Springer-Verlag Berlin Heidelberg 2014

\begin{abstract}
In recent years, diabetes mellitus has become an epidemic and now represents one of the most prevalent disorders. Cardiovascular complications are the major cause of mortality and morbidity in diabetic patients. While ischaemic events dominate the cardiac complications of diabetes, it is widely recognised that the risk for developing heart failure is also increased in the absence of overt myocardial ischaemia and hypertension or is accelerated in the presence of these comorbidities. These diabetes-associated changes in myocardial structure and function have been called diabetic cardiomyopathy. Numerous molecular mechanisms have been proposed to contribute to the development of diabetic cardiomyopathy following analysis of various animal models of type 1 or type 2 diabetes and in genetically modified mouse models. The steady increase in reports presenting novel mechanistic data on this subject expands the list of potential underlying mechanisms. The current review provides an update on molecular alterations that may contribute to the structural and functional alterations in the diabetic heart.
\end{abstract}

Keywords Animal models · Autophagy · Cardiomyopathy · Diabetes $\cdot$ Fibrosis $\cdot$ Heart failure $\cdot$ Inflammation $\cdot$

Lipotoxicity $\cdot$ Metabolism $\cdot$ Review

$\begin{array}{ll}\text { Abbreviations } \\ \text { AIF } \quad \text { Apoptosis-inducing factor } \\ \text { AMPK } & \text { AMP-activated protein kinase }\end{array}$

H. Bugger

Cardiology and Angiology I, Heart Center Freiburg University,

Freiburg, Germany

\section{E. D. Abel $(\bowtie)$}

Fraternal Order of Eagles Diabetes Research Center, Division of Endocrinology and Metabolism, Roy J. and Lucille A. Carver College of Medicine, University of Iowa, 108 CMAB, 451 Newton

Road, Iowa City, IA 52242-1101, USA

e-mail: DRCAdmin@uiowa.edu

$\begin{array}{ll}\text { CAD } & \text { Coronary artery disease } \\ \text { CIRKO } & \text { Cardiomyocyte insulin receptor knockout } \\ \text { CTGF } & \text { Connective tissue growth factor } \\ \text { DGAT } & \text { Diacylglycerol acyl transferase } \\ \text { ER } & \text { Endoplasmic reticulum } \\ \text { FA } & \text { Fatty acid } \\ \text { FOXO1 } & \text { Forkhead box O1 } \\ \text { GRP } & \text { Glucose-regulated protein } \\ \text { HDAC } & \text { Histone deacetylase } \\ \text { LC3 } & \text { Microtubule-associated protein 1A/1B-light chain 3 } \\ \text { LPL } & \text { Lipoprotein lipase } \\ \text { LV } & \text { Left ventricular } \\ \text { MAPK } & \text { Mitogen-activated protein kinase } \\ \text { MHC } & \text { Myosin heavy chain } \\ \text { miRNA } & \text { MicroRNA } \\ \text { MMP } & \text { Matrix metalloproteinase } \\ \text { MTOR } & \text { Mammalian target of rapamycin } \\ \text { MVO } & \text { Myocardial oxygen consumption } \\ \text { NF-KB } & \text { Nuclear factor } \text { kB } \\ \text { OXPHOS } & \text { Oxidative phosphorylation } \\ \text { PARP-1 } & \text { Poly(ADP-ribose) polymerase-1 } \\ \text { PI3K } & \text { Phosphatidylinositol 3-kinase } \\ \text { RAAS } & \text { Renin-angiotensin-aldosterone system } \\ \text { RAGE } & \text { Receptor for AGEs } \\ \text { ROS } & \text { Reactive oxygen species } \\ \text { SERCA } & \text { Sarcoplasmic/endoplasmic reticulum calcium } \\ & \text { ATPase } \\ \text { SR } & \text { Sarcoplasmic reticulum } \\ \text { STZ } & \text { Streptozotocin } \\ \text { UPR } & \text { Unfolded protein response } \\ & \end{array}$

\section{Introduction}

The prevalence of diabetes mellitus continues to increase and is driven in part by the accompanying obesity epidemic. In 2011, 
$8.3 \%$ of the US population or 25.8 million individuals have diabetes (18.8 million people diagnosed and 7.0 million people undiagnosed). In addition, $25.4 \%$ are considered to have prediabetes [1]. Cardiovascular complications, mainly ischaemic heart disease, are the major cause of morbidity and mortality in patients with diabetes. However, in population-based studies, the risk of heart failure in patients with diabetes (particularly type 2) is significantly increased following adjustment for wellestablished heart failure risk factors such as hypertension or ischaemic heart disease. The earliest report of this association was published 40 years ago by Rubler and colleagues, who reported post-mortem data from four diabetic patients following death from heart failure without evidence of hypertension, myocardial ischaemia or congenital or valvular heart disease [2]. Similarly, a more recent study by de Simone et al reported an increased risk of heart failure in patients with type 2 diabetes, independent of concurrent myocardial infarction and hypertension [3]. Thus, the term 'diabetic cardiomyopathy' was coined, defined as ventricular dysfunction in the absence of coronary artery disease (CAD) and hypertension. Some have argued that if a strict definition of structural changes leading to clinically meaningful cardiac dysfunction is applied, then a distinct cardiomyopathy of diabetes might not exist in humans with type 1 diabetes [4]. Nevertheless, many studies in humans have provided strong evidence for structural and subcellular changes that adversely affect the response of the heart to subsequent stressors. Potential mechanisms extend beyond consequences of diabetesassociated hyperglycaemia and reflect the interaction of multiple factors, which in combination may adversely affect cardiac structure and function. The current review provides an overview of recently described molecular mechanisms that may contribute to the development of diabetic cardiomyopathy.

\section{Structural and functional changes}

A prominent feature of the diabetic myocardium is cardiac hypertrophy. Data from the Strong Heart study and the Cardiovascular Health study revealed an independent association between diabetes and cardiac hypertrophy (increased left ventricular [LV] mass and wall thickness) that was accompanied by compromised systolic and diastolic function $[5,6]$. These changes in myocardial structure were confirmed independently in the Framingham cohort where cardiac hypertrophy was noted to correlate with glycaemic control in diabetic women independently of blood pressure [7].

Diastolic dysfunction has been described as an early functional alteration in the diabetic myocardium. Using conventional echocardiography and tissue Doppler imaging, diastolic dysfunction is detected in $40-75 \%$ of patients with type 1 or type 2 diabetes $[8,9]$. Systolic dysfunction may also develop but mostly at a later stage of the disease, presenting a challenge for diagnosis using conventional echocardiography. Using strain analysis and measurements of peak systolic velocity, subtle abnormalities in systolic function have, however, been described in $24 \%$ of patients with diabetes mellitus without CAD or LV hypertrophy [10]. A recent study showed that using systolic strain analyses, systolic dysfunction can also be detected in diabetic patients with normal diastolic function, suggesting that diastolic dysfunction may not necessarily be the first functional alteration in diabetic cardiomyopathy [11].

Systolic and diastolic dysfunction can be consistently reproduced in a variety of rodent models, including models of type 1 and type 2 diabetes, as reviewed in detail elsewhere [12]. Indeed, mice and rats represent a useful model system with which to investigate molecular mechanisms of diabetic cardiomyopathy since rodents do not develop atherosclerosis when rendered diabetic in the absence of mutations that precipitate severe hypercholesterolaemia [13].

Diabetes increases the risk of heart failure. The increased heart failure risk persists even after adjusting for underlying cardiac ischemia and hypertension. Diabetes is associated with detectable changes in LV structure and function in the absence of active ischaemia.

\section{Molecular mechanisms of diabetic cardiomyopathy}

In the following subsections, we will provide concise overviews of molecular mechanisms that have been proposed to contribute to the development of diabetic cardiomyopathy, with a focus not only on established mechanisms but also on novel or emerging mechanisms (summarised in Fig. 1). Many of these areas warrant independent and comprehensive review. Because of space limitations our intent is to provide the reader with an introduction to the complex pathophysiology of diabetic cardiomyopathy as a road map for more detailed exploration. In addition, it is important to note that many of these mechanisms are highly interrelated, despite being presented as separate topics. Examples of these interactions are mentioned in the legend to Fig. 1.

The metabolic milieu associated with diabetes, such as hyperglycaemia, increased circulating fatty acids and triacylglycerols, hyperinsulinaemia, increased inflammatory cytokines, alter multiple molecular pathways within the cardiomyocyte, which impair cardiac contractility and promote myocyte dysfunction, injury and cell death.

AGES AGEs are predominantly long-lived proteins that become glycated after exposure to sugars, which alters their functional properties [14]. The increased formation of AGEs secondary to 


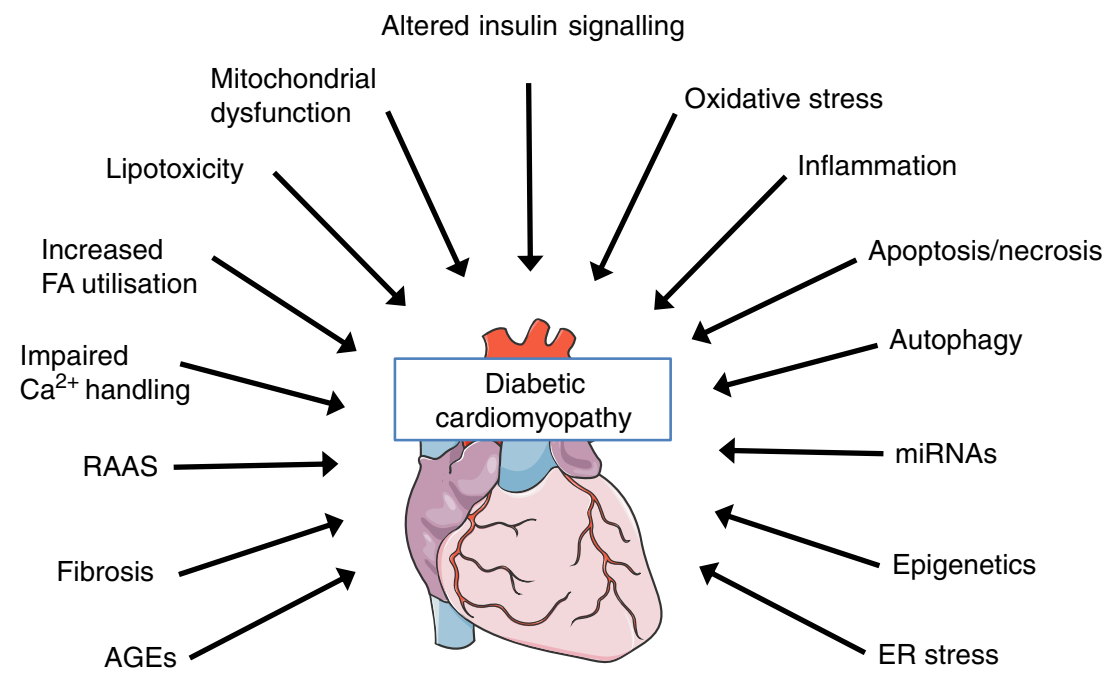

Fig. 1 Schematic depiction of the multiple potential mechanisms that have been implicated in the pathophysiology of diabetic cardiomyopathy. Although depicted as separate mechanisms, these pathways interact with each other in complex ways. Examples include: (1) increased FA

hyperglycaemia may alter structural proteins and lead to increased myocardial stiffness. AGEs can cause crosslinks in collagen molecules, thereby impairing the ability of collagen to be degraded, leading to increased fibrosis with subsequent increased myocardial stiffness and impaired cardiac relaxation [15]. AGEs also act via AGE receptors (RAGE), the expression of which is induced in diabetic hearts by oxidative stress [16]. Increased AGE and RAGE activation leads to activation of nuclear factor $\mathrm{kB}(\mathrm{NF}-\mathrm{kB})$ signalling, which may contribute to the switch towards increased expression of the $\beta$-myosin heavy chain (MHC) isoform in diabetic hearts [16]. Treatment with dehydroepiandrosterone counteracts oxidative stress-induced RAGE activation both in hearts of streptozotocin (STZ)-induced diabetic rats and Zucker diabetic fatty (ZDF) rats and normalises $\mathrm{NF}-\mathrm{k} \beta$ signalling and the MHC isoform switch, which are early events in diabetic cardiomyopathy. In addition, hearts of STZdiabetic rats exhibit crosslinked AGEs on the sarcoplasmic/ endoplasmic reticulum calcium ATPase (SERCA)-2a pump, which may impair sarcoplasmic reticulum (SR) $\mathrm{Ca}^{2+}$ reuptake in cardiac myocytes [17, 18]. Long-term treatment with an AGE crosslink breaker partially normalises $\mathrm{SR} \mathrm{Ca}^{2+}$ handling [18]. In a separate study, STZ-induced diabetes was observed to markedly decrease the maximum and minimum rate of pressure change in the ventricle and LV developed pressure determined by cardiac catheterisation. These haemodynamic impairments were prevented by RAGE gene knockdown [19]. Thus, targeting the AGE/RAGE axis may represent a potentially useful therapeutic approach that needs to be explored in further detail.

Fibrosis Increased fibrosis is frequently observed in diabetic cardiomyopathy. Increased perivascular and intermyofibrillar fibrosis has been observed in human myocardial samples in the absence of CAD and hypertension [20, 21]. Similarly, in oxidation and lipotoxicity may promote mitochondrial dysfunction; (2) mitochondrial dysfunction and ER stress may increase apoptosis; (3) oxidative stress, increased AGE signalling and inflammation may promote increased expression of pro-fibrotic genes or promote apoptosis

rodent models, hearts of rodents with STZ-induced type 1 diabetes and Otsuka Long Evans Tokushima fatty (OLETF) rats with type 2 diabetes show increased connective tissue content [22-24]. Increased collagen deposition may be related to increased expression of TGF- $\beta$ and connective tissue growth factor (CTGF), transcription factors that drive collagen production, or may be related to increased activation of poly (ADP-ribose) polymerase 1 (PARP-1) [25]. In addition, dysregulation of extracellular matrix degradation due to remodelling of matrix metalloproteinases (MMPs), in particular reduced expression of MMP-2, contribute to increased connective tissue content in diabetic hearts $[22,26]$.

Inflammation Diabetes is a pro-inflammatory state [27] and many groups have reported an increase in tissue concentrations of cytokines in various mouse models of diabetes suggesting an important contribution of inflammation to the development of diabetic cardiomyopathy. These studies demonstrated intramyocardial inflammation in diabetic cardiomyopathy including increased expression of cell adhesion molecules (ICAM-1 and vascular cell adhesion molecule 1 [VCAM-1]), increased infiltration of macrophages and leucocytes and increased expression of inflammatory cytokines (IL-1 $\beta$, IL-6, IL-18, TNF- $\alpha$ and TGF- $\beta 1$ ) [22, 28-31]. Many interventions have demonstrated beneficial effects, attributable in part to reduced cardiac inflammation. These interventions include angiotensin 1 (AT-1) receptor antagonism, activation of the kallikreinkinin system, inhibition of $\mathrm{p} 38$ mitogen-activated protein kinase (MAPK) signalling, gene deletion of kinin receptor b1, inhibition of interleukin converting enzyme, atorvastatin treatment, anti-TNF- $\alpha$ treatment, inactivation of GSK- $3 \beta$, and cannabidiol treatment [22, 26, 28-30, 32-35]. Other proposed mechanisms for increased inflammation in diabetic cardiomyopathy include 
oxidative stress via a Ras-related C3 botulinum toxin substrate 1 (RAC1)-mediated activation of NADPH oxidase and endoplasmic reticulum (ER) stress [36]. Although most of these mechanisms have been studied in animal models of insulin-deficient (type 1) diabetes, a smaller number of studies in animal models of type 2 diabetes, such as the Zucker diabetic rat [37], the lowdose STZ and high-fat diet rat model [38] or mice with dietinduced obesity and diabetes [39], also revealed increased myocardial inflammation that was driven in part by increased activation of M1 macrophages. Data on myocardial inflammation in humans with diabetes are lacking to date. However, the findings in animal models provide a strong rationale for studying these mechanisms in humans, to definitively determine whether antiinflammatory strategies will have any clinical usefulness in minimising cardiac complications of diabetes.

Apoptotic and necrotic cell death Many of the molecular mechanisms that increase fibrosis and myocardial inflammation can activate pro-apoptotic signalling pathways or activate necrosis signalling. Increased cell death is frequently observed in hearts of humans with type 1 and type 2 diabetes and rodent models of these disorders, and includes both apoptotic and necrotic cell death [40-44]. Underlying mechanisms for increased rates of apoptosis include increased production of reactive oxygen species (ROS), increased circulating inflammatory cytokines and chemokines, caspase activation, Fasreceptor-dependent and mitochondrion-dependent apoptosis, ER stress, increased activation of the TGF- $\beta$ signalling pathway, increased local activation of the renin-angiotensin-aldosterone system (RAAS), IGF-1 resistance and altered expression of pro- and anti-apoptotic molecules [42-48]. Increased PARP-1 activation, impaired IGF-1 action and increased angiotensin II levels may also promote necrosis in diabetic hearts [48]. Since overactivation of PARP-1 triggers the nuclear translocation of apoptosis-inducing factor (AIF), resulting in caspase-independent cell death, PARP-1-mediated cell death may be distinct from apoptosis, necrosis or also autophagy and is therefore termed parthanatos [49]. Recent data suggest a role for forkhead box O1 (FOXO1)-mediated inducible nitric oxide synthase-induced S-nitrosylation of PARP-1 target proteins, such as glyceraldehyde 3-phosphate dehydrogenase and caspase-3, in initiating cardiac cell death in diabetic cardiomyopathy [50].

$R A A S$ Activation of the intrinsic myocardial RAAS promotes remodelling processes in the heart. Antagonising myocardial aldosterone action has beneficial effects on cardiac myocyte hypertrophy and myocardial fibrosis [51]. Several studies have demonstrated increased connective tissue content in STZ-diabetic mouse hearts, which can be attenuated by treatment with the aldosterone antagonist spironolactone [22, 23, 26]. Cardiac angiotensin II receptor density and synthesis is increased in STZ hearts, and increased superoxide production and apoptosis can also be inhibited, at least partially, by treatment with angiotensin receptor blockers or ACE inhibitors [23, 52].

Impaired $\mathrm{Ca}^{2+}$ handling Excitation contraction coupling is essential for cardiac contractility. Excitation of the cardiomyocyte results in increased $\mathrm{Ca}^{2+}$ influx via L-type $\mathrm{Ca}^{2+}$ channels, which in turn triggers the release of $\mathrm{Ca}^{2+}$ from the SR, resulting in actin-myosin interaction and mechanical work. Relaxation occurs when $\mathrm{Ca}^{2+}$ is actively re-imported into the SR by SERCA-2a. In cardiomyocytes from an $o b / o b$ mouse model of type 2 diabetes, intracellular resting $\mathrm{Ca}^{2+}$ concentrations were elevated, intracellular $\mathrm{Ca}^{2+}$ decay was prolonged, $\mathrm{Ca}^{2+}$ transients were slower and smaller, responsiveness to extracellular $\mathrm{Ca}^{2+}$ was diminished, SERCA 2a activity was decreased and SR $\mathrm{Ca}^{2+}$ reuptake was impaired [53-55]. Perturbations in cardiac $\mathrm{Ca}^{2+}$ handling have also been found in Type 2 diabetic $d b / d b$ mice, including decreased systolic and diastolic levels of $\mathrm{Ca}^{2+}$, decreased rates of $\mathrm{Ca}^{2+}$ decay, $\mathrm{Ca}^{2+}$ leakage from the SR and decreased SR $\mathrm{Ca}^{2+}$ load [56, 57]. Impairment in intracellular $\mathrm{Ca}^{2+}$ handling in the heart also occurs in rodent models of type 1 diabetes, involving increased resting $\mathrm{Ca}^{2+}$ levels, attenuated $\mathrm{SR} \mathrm{Ca}^{2+}$ release and reuptake, delayed recovery of the intracellular $\mathrm{Ca}^{2+}$ transient, reduced expression of SERCA- $2 \mathrm{a}$ and the $\mathrm{Na}^{+}-\mathrm{Ca}^{2+}$ exchanger and compromised mitochondrial $\mathrm{Ca}^{2+}$ handling [12, 58-63]. Very recent studies have implicated diabetesinduced or glucose-dependent modifications of $\mathrm{Ca}^{2+} /$ calmodulin-dependent protein kinase 2 (CAMKII) (i.e. oxidation or modifications by $O$-linked $N$-acetylglucosamine), which in diabetic models will impair SERCA function or ryanodine receptor function, linking glucose-derived modifications of this important signalling molecule to the pathophysiology of contractile dysfunction in diabetes [64-66].

Increased fatty acid utilisation Hearts in humans with type 1 and type 2 diabetes and in rodent models of type 1 and type 2 diabetes are characterised by increased fatty acid (FA) uptake and oxidation [67-69]. Increased FA utilisation likely results from increased serum FA and triacylglycerol levels, and increased activity of peroxisome proliferator-activated receptor $\alpha(\operatorname{PPAR} \alpha)$, which determines FA oxidative capacity by increasing FA oxidation gene expression [70]. Concomitantly, glucose uptake, glycolysis and glucose oxidation are reduced in diabetic hearts, most likely mediated by decreased GLUT4 expression and translocation and impaired pyruvate decarboxylation [71-73]. Increased FA oxidation in diabetic hearts has been shown to be associated with increased myocardial oxygen consumption $\left(\mathrm{MVO}_{2}\right)$, which is not accompanied by an equivalent increase in cardiac contractility and thus results in reduced cardiac efficiency (cardiac work/ $\mathrm{MVO}_{2}$ ) [68, 74]. Increased $\mathrm{MVO}_{2}$ and decreased cardiac efficiency is likely the consequence of FA-induced mitochondrial uncoupling of 
ATP synthesis from oxygen consumption, leading to energy depletion $[75,76]$. This uncoupling is mediated by increased proton flux through mitochondrial uncoupling proteins, which are activated in this setting by reactive oxygen radicals $[75$, 77]. Interestingly, FA-induced ROS-mediated mitochondrial uncoupling does not occur in certain mouse models of type 1 diabetes, such as the Akita diabetic mouse, indicating that some mechanisms governing changes in myocardial energy metabolism might be distinct in type 1 vs type 2 diabetes [67].

Lipotoxicity Diabetes is associated with myocardial lipid accumulation. Cardiac lipotoxicity involves detrimental effects that may arise from increased cardiomyocyte lipid uptake, use and storage, which may adversely impact cardiomyocyte function. It is likely that the mechanism for lipotoxicity arises not from accumulation of triacylglycerol per se but is a result of increased availability of lipid intermediates such as ceramides, diacylglycerol or oxidised phospholipids. Lipotoxic cardiomyopathy has been modelled in various transgenic animals in which FA uptake was increased, including overexpression of long-chain acyl-CoA synthetase 1, a glycosylphosphatidylinositol membrane-anchored form of lipoprotein lipase (LPL), or FA transport protein 1 [78-80]. Increased myocardial lipid utilisation and storage is also observed in literally all animal models of diabetes to varying extents and in human cardiac tissue as well [12, 69, 81]. Importantly, multiple approaches that either increase triacylglycerol formation (diacylglycerol acyl transferase transgenic) or increase FA oxidation [82] or increase lipid export (ApoB transgenics) or decrease lipid uptake (CD36 or LPL knockout), in addition to anti-steatotic therapy with thiazolidinediones, leptin or alpha lipoic acid can normalise lipid-induced cardiac dysfunction [83-85]. These observations suggest that increased utilisation or increased sequestration of lipids may reduce the availability of potentially toxic lipid intermediates. An important underlying mechanism for lipotoxic damage may be increased rates of apoptotic cell death, which may be due to increased ceramide biosynthesis, increased ROS production, remodelling of the mitochondrial membrane phospholipid composition, including a decrease in cardiolipin content, and increases in the saturated lipid content of the ER resulting in ER stress [86-89]. A recent study by Michel et al also implicates small nucleolar RNAs (snoRNAs) in the lipotoxic response of Chinese hamster ovary cells to palmitate-induced apoptosis [90]. Finally, increased ceramide levels can also activate inflammatory signalling pathways and increase reactive nitrogen species [83].

Mitochondrial dysfunction Mitochondrial dysfunction has been implicated in the pathogenesis of diabetes and its complications in literally all diabetes-affected tissues. The most compelling evidence for mitochondrial dysfunction in human diabetic hearts was recently provided by Anderson and colleagues, who observed impaired mitochondrial respiratory capacity, evidence of increased mitochondrial oxidative stress, and increased sensitivity to calcium-induced opening of the mitochondrial permeability transition pore in atrial tissue of patients with type 2 diabetes [91, 92]. Substantial evidence of mitochondrial dysfunction in diabetic hearts already existed 30 years ago when Kuo and colleagues demonstrated reduced state 3 respiration of mitochondria isolated from hearts of obese and Type 2 diabetic $d b / d b$ mice [93]. Impairment in state 3 respiration, mitochondrial oxidative stress and abnormal mitochondrial ultrastructure have been observed in various rodent models with different degrees of obesity, insulin resistance and insulindependent and non-insulin-dependent diabetes (for detailed review, see [12]). Mechanisms underlying the impairment in oxidative capacity and mitochondrial morphology include oxidative damage, transcriptional and translational alterations of oxidative phosphorylation (OXPHOS) subunit expression, impaired mitochondrial calcium handling and changes in cardiac insulin signalling (for detailed review see $[63,94]$ ). Mitochondrial dysfunction in diabetic hearts also includes FAinduced mitochondrial uncoupling, which is described above (see 'Increased fatty acid utilisation').

Altered myocardial insulin signalling Rodents in models of type 2 diabetes exhibit insulin resistance in multiple tissues, including the heart $[68,74]$. However, it is important to note that whereas impaired insulin-mediated glucose uptake is nearly universally observed, activation of proximal insulin signalling pathways may persist in humans and rodents with diet-induced obesity and type 2 diabetes [73, 95]. Indeed, excessive myocardial insulin signalling might contribute to LV dysfunction in pressure overload cardiac hypertrophy [96] and prevent ischaemic preconditioning [97]. Conversely, prolonged high-fat feeding impairs downstream myocardial insulin signalling to Akt and FOXO1, which may also independently contribute to cardiac dysfunction [98]. Using mice with a cardiomyocyte-restricted deletion of the insulin receptor (CIRKO mice), we reported that complete absence of myocardial insulin signalling impairs cardiac contractility, mitochondrial respiratory capacity and expression of OXPHOS genes [99]. In addition, mitochondrial levels of proteins involved in FA oxidation and the tricarboxylic acid cycle were decreased, and remodelling of the OXPHOS subunit composition occurs at the protein level. Importantly, CIRKO mice exhibit FA-induced ROS-mediated mitochondrial uncoupling, as observed in models of type 2 diabetes, and the presence of mitochondrial defects caused by cardiac insulin resistance may contribute to impaired cardiac efficiency following induction of diabetes [99, 100].

Oxidative stress Oxidative stress is widely accepted to play an important role in the development and progression of diabetes and its complications. ROS can directly damage proteins or phospholipids by oxidation, or secondarily, by oxidising lipids 
to reactive lipid peroxides, or by generating reactive nitrogen species from nitric oxide. DNA is another major site of ROSinduced damage and mitochondrial DNA has been proposed to be particularly susceptible to oxidative damage [101]. Evidence of increased oxidative stress in human diabetic hearts has been provided by Anderson et al who demonstrated increased mitochondrial $\mathrm{H}_{2} \mathrm{O}_{2}$ emission and increased levels of 4-hydroxynonenal-modified proteins and 3-nitrotyrosinemodified proteins [91]. In animal models of diabetes, several independent groups reported increased mitochondrial protein tyrosine nitration, increased levels of lipid peroxidation products, decreased levels of reduced glutathione and induction of the antioxidant defence system $[59,75,102,103]$. More causal evidence for a role of ROS in the pathogenesis of diabetic cardiomyopathy comes from studies by Epstein and colleagues, who demonstrated that transgenic overexpression of catalase or manganese superoxide dismutase in diabetic mice at least partially restored impaired mitochondrial function and cardiomyocyte contractility $[59,104]$. Interestingly, although short-term incubation of cardiomyocytes from non-diabetic control animals under high glucose conditions did not increase ROS production, similar treatment of cardiomyocytes isolated from the OVE26 mouse model of type 1 diabetes induced ROS overproduction. A mitochondrial source for these ROS was confirmed by overexpression of catalase or by inhibition of OXPHOS complex I or II. Thus diabetes-induced changes in mitochondria increase their propensity to overproduce superoxide, which is exacerbated in a hyperglycaemic environment. Further evidence for a mitochondrial source of ROS in diabetic hearts comes from Abel's group who directly demonstrated increased production of mitochondrial superoxide in diabetic $d b / d b$ mouse hearts [75]. Interestingly, some models of type 1 diabetes do not exhibit increased mitochondrial superoxide generation in the heart, including the Akita mouse model and STZ-induced diabetes, suggesting that mitochondria might not be the major or sole source of ROS across multiple diabetes models, or that certain mechanisms are distinct between animal models of type 1 and type 2 diabetes [67, 105]. Others have also shown increased myocardial NADPH oxidase-derived ROS production in STZ-induced diabetes, $o b / o b$ mice and obese Zucker falfa rats; this is consistent with the model that oxidative stress in diabetic hearts results both from mitochondrial and extramitochondrial sources [35, 42, 106, 107].

Autophagy An interesting novel, but as yet relatively underinvestigated, candidate mechanism that may potentially contribute to the pathophysiology of diabetic cardiomyopathy is autophagy. Autophagy is a physiological process by which long-lived proteins, ribosomes, lipids and even entire cellular organelles are engulfed by double-membrane structures, which are subsequently targeted to lysosomes for degradation [108]. A low level of constitutive autophagy is important in the heart for maintaining normal cellular function and protein and organellar quality control [109]. Defects in this process lead to cardiac dysfunction and heart failure, particularly when cellular stress is increased [109]. Autophagy is implicated in various cardiac disease states, including ischaemia-reperfusion, chronic ischaemia, cardiac hypertrophy and heart failure [110-112]. Autophagy induction can ameliorate disease pathogenesis or contribute to the progression of disease as a function of the context or magnitude of induction $[34,113]$. For example, activation of autophagy is protective during ischaemia, when energy depletion occurs [112], whereas it may be maladaptive in the load-stressed heart and during postischaemic reperfusion [111, 112].

Little information is available regarding a role for autophagy in the pathophysiology of diabetic cardiomyopathy. However, recent findings have provided compelling evidence that insulin signalling is an important regulator of myocardial autophagy $[114,115]$. Feeding mice a $60 \%$ fructose diet for 12 weeks to induce type 2 diabetes caused myocardial insulin resistance, which was associated with accumulation of autophagosomes as demonstrated by increased levels of the autophagic markers LC3B (microtubule-associated protein 1A/1B-light chain 3) and p62 (nucleoporin p62) [116]. The concomitant increase in p62 raises the possibility that turnover of autophagosomes could be impaired in this model. Phosphatidylinositol 3-kinase (PI3K)/Akt signalling can negatively regulate autophagy by inhibiting the mammalian target of rapamycin (MTOR) and, accordingly, changes in autophagy were associated with reduced PI3K/Akt signalling in these insulin-resistant hearts, raising the possibility that myocardial insulin resistance may trigger autophagy on the basis of reduced PI3K/Akt signalling [117]. These results could imply a detrimental effect of autophagy induction in insulin-resistant hearts. Conversely, autophagy induction in ischaemic hearts has been proposed to play a cardioprotective role $[112,118,119]$. How do we reconcile the potential paradox that autophagy induction, while being beneficial in ischaemic hearts, could be detrimental in diabetic hearts? First, it is important to note that it remains to be determined whether autophagy induction in hearts of models of obesity and insulin resistance represents a compensatory adaptation or directly contributes to pathophysiology. There is an emerging consensus that induction of autophagy can either antagonise disease pathogenesis or contribute to the progression of disease depending on the context and amplitude of the induction [120]. Keeping this in mind, it can be speculated that acute autophagy induction (e.g. ischaemic heart) might be beneficial whereas persistent autophagy induction (e.g. diabetic heart, or as occurs in the failing heart) could be detrimental, $[111,121]$. Thus, the question of whether or not autophagy induction is beneficial or maladaptive in insulin-resistant hearts remains to be clarified using appropriate mechanistic models.

Xie et al demonstrated that in models of type 1 diabetes (STZ model or OVE26 mice) cardiac dysfunction was associated with repression of myocardial autophagy. This was 
hypothesised to occur on the basis of impaired activation of the energy metabolic sensor AMP-activated protein kinase (AMPK) [122]. AMPK activation inhibits MTOR and can therefore activate autophagy [112]. Thus, restoration of AMPK activity by metformin treatment enhanced autophagic activity and improved cardiac function, suggesting that the reduction in autophagy may have contributed to the disease development [123]. Therefore, hearts in type 1 and type 2 diabetes may differ in autophagy activation (decreased vs increased) and in the mechanisms that regulate autophagy (PI3K/Akt vs AMPK). More studies are required to elucidate whether autophagy is beneficial or detrimental in diverse models of diabetes, whether regulatory mechanisms are identical or different and whether and how the severity and duration of diabetes might contribute to the changes in autophagy observed in diabetic hearts.

MicroRNAs Diabetic cardiomyopathy is associated with changes in global patterns of gene expression, which are associated with microRNAs (miRNAs). These regulators of gene expression are endogenous, noncoding, single-strand RNAs with an average length of 22 nucleotides and are encoded by short inverted repeats within the genome. miRNAs regulate gene expression by two mechanisms, namely repression of translation or by promoting degradation of target mRNAs. Changes in individual miRNA levels have been demonstrated to play important roles in the pathogenesis of a variety of diseases including diabetes. Recent reports suggested a role for miR-143, miR-181, miR-103, miR-107 and miR-802 in the regulation of systemic glucose metabolism and insulin sensitivity, thereby implicating miRNAs in the pathogenesis of insulin resistance and type 2 diabetes [124-126].

A change in myocardial miRNA content is a plausible mechanism that could be linked to changes in cardiac function. Global dysregulation of the miRNA biogenesis machinery in the heart by cardiomyocyte-specific deletion of dicer leads to rapidly progressive dilated cardiomyopathy and heart failure [127]. Recent studies have also linked dysregulation of specific miRNAs to the pathophysiology of diabetic cardiomyopathy $[128,129]$. MicroRNA-1, which accounts for approximately $40 \%$ of the total myocardial miRNA pool, has been shown to downregulate Pim-1 in STZ-induced Type 1 diabetic mice, and restoration of Pim-1 levels prevented cardiomyocyte apoptosis, ventricular dilatation and failure [130]. Myocardial expression of miRNA-133 is increased in the alloxan-induced rabbit model of type 1 diabetes, and miRNA-133 modulates connective tissue content by regulating CTGF expression, suggesting its contribution to fibrosis induction in diabetic hearts $[131,132]$. Considering that the human genome may encode for more than 1,000 miRNAs and that more than $60 \%$ of all mammalian genes may represent conserved targets of miRNAs, and considering convincing data supporting a role for specific miRNAs in the regulation of systemic metabolism, it appears likely that future studies will identify novel dysregulation of additional specific miRNAs that may contribute to the molecular defects that characterise diabetic cardiomyopathy [133, 134].

Epigenetics Epigenetics refers to inheritable changes in gene expression patterns that are not associated with altered DNA sequence. Histone modification, in particular histone acetylation, is a major epigenetic mechanism that regulates gene expression. The equilibrium of histone acetylation is largely maintained through functional interplay between histone acetyltransferases, which catalyse histone acetylation, and histone deacetylases (HDACs), which remove acetyl groups. Dysregulation of histone acetylation contributes to the development of a variety of diseases, and HDAC inhibition is a widely discussed therapeutic option to potentially treat a multitude of prevalent diseases such as cancer and neurodegenerative, inflammatory and cardiovascular disorders [135-137].

HDACs can be categorised into four classes depending on sequence identity and domain organisation. Class II HDACs (HDAC5, HDAC9) are able to suppress cardiac hypertrophy, while class I HDACs promote cardiac hypertrophy $[138,139]$. In hearts of Type 2 diabetic $d b / d b$ mice, renal failure induced by unilateral nephrectomy increased myocardial acetylation of histone 3 at lysine 23 and 9, which correlated with increased expression of cardiomyopathy-related genes and cardiac hypertrophy [140]. These observations suggest an interaction between uraemia and cardiac hypertrophy in type 2 diabetes via a mechanism mediated by epigenetic modifications of histone $\mathrm{H} 3$ in cardiomyocytes.

Another well-recognised epigenetic regulator is methylation of nuclear DNA. DNA methylation occurs mainly on $\mathrm{CpG}$ islands in the $5^{\prime}$ regulatory regions of many genes. In many disease processes, such as cancer, $\mathrm{CpG}$ islands in gene promoters acquire abnormal hypermethylation, resulting in transcriptional silencing. Using cardiomyocytes of STZdiabetic rats, Monkemann et al demonstrated that overexpression of the gene encoding p21 and inhibition of expression of the gene encoding cyclin D1 in diabetic hearts are regulated by demethylation and hypermethylation, respectively, of their 5'-flanking regions upon development of diabetes [141]. The proteins are involved in cell cycle regulation and are induced or repressed in diabetic tissues [142, 143]. Taken together, epigenetic modifications, which have the potential to influence the expression of the entire genome, may represent an under-investigated mechanism, which may potentially contribute to the pathogenesis of diabetic cardiomyopathy.

ER stress A major function of the ER is protein folding and modification. These processes can be interrupted by various 
factors, such as oxidative stress or perturbed calcium homeostasis, leading to accumulation of unfolded proteins, which ultimately end in apoptotic cell death. This fate can be prevented by a quality control mechanism termed unfolded protein response (UPR), which increases the levels of chaperones and related proteins that accomplish protein folding in the ER. ER stress has been identified to contribute to myocardial apoptosis in animal models of type 1 and type 2 diabetes, as demonstrated by induction of UPR signalling proteins and ER stress-related apoptotic signalling proteins such as glucose-regulated protein (GRP)78, GRP94, cleaved activating transcription factor 6 (ATF6), phosphorylated eIF2 $\alpha$, C/EBP homologous protein (CHOP) and caspase 12 [144-146]. Several studies suggest that ER stress may be mediated by increased oxidative stress in diabetic cardiomyopathy [36, 145, 147]. Of interest, while GLP-1 agonism may affect the diverse mechanisms that are implicated in the pathophysiology of diabetic cardiomyopathy, ER stress was recently noted to be attenuated by this treatment $[148,149]$.

Multiple molecular mechanisms act in concert to impair cardiac function and promote cardiomyocyte injury in diabetes:

- Altered signal transduction (insulin signalling, reninangiotensin signalling)

- Altered metabolism and mitochondrial dysfunction

- Post-translational modifications of structural and signalling proteins

- Altered cell homeostatic processes such as apoptosis and autophagy and ER stress

- Changes in gene regulation (activation of transcription factors, microRNAs and epigenetic mechanisms)

\section{Conclusions}

A large body of evidence supports the existence of a diabetic cardiomyopathy, which will increase in prevalence in parallel with the obesity epidemic. The increased vulnerability of individuals with diabetes to cardiovascular complications and heart failure underscores the importance of developing novel therapeutic approaches. Many potential mechanisms have been proposed and studied, and some interventions have demonstrated beneficial effects on the associated pathological features of diabetic cardiomyopathy in preclinical models. Some mechanisms, such as autophagy, microRNAs and epigenetic mechanisms, remain relatively under-investigated and require further study. Thus an important need remains to further delineate the basic mechanisms of diabetic cardiomyopathy and to translate promising therapies in preclinical models to humans.

Funding HB is supported by the German Research Foundation (DFG) and EDA by the National Institutes of Health $(\mathrm{NIH})$.

Duality of interest The authors declare that there is no duality of interest associated with this manuscript.

Contribution statement $\mathrm{HB}$ and EDA were responsible for the conception and design of the manuscript, drafting the article and revising it critically for important intellectual content. Both authors approved the version to be published.

\section{References}

1. American Diabetes Association (2011) Diabetes statistics. www. diabetes.org/diabetes-basics/diabetes-statistics/. Accessed 25/12/ 2013.

2. Rubler S, Dlugash J, Yuceoglu YZ, Kumral T, Branwood AW, Grishman A (1972) New type of cardiomyopathy associated with diabetic glomerulosclerosis. Am J Cardiol 30:595-602

3. de Simone G, Devereux RB, Chinali M et al (2010) Diabetes and incident heart failure in hypertensive and normotensive participants of the Strong Heart Study. J Hypertens 28:353-360

4. Litwin SE (2013) Diabetes and the heart: is there objective evidence of a human diabetic cardiomyopathy? Diabetes 62:3329-3330

5. Devereux RB, Roman MJ, Paranicas M et al (2000) Impact of diabetes on cardiac structure and function: the Strong Heart Study. Circulation 101:2271-2276

6. Lee M, Gardin JM, Lynch JC et al (1997) Diabetes mellitus and echocardiographic left ventricular function in free-living elderly men and women: the Cardiovascular Health Study. Am Heart J 133:36-43

7. Kannel WB, McGee DL (1979) Diabetes and cardiovascular disease. The Framingham study. JAMA 241:2035-2038

8. Boyer JK, Thanigaraj S, Schechtman KB, Perez JE (2004) Prevalence of ventricular diastolic dysfunction in asymptomatic, normotensive patients with diabetes mellitus. Am J Cardiol 93:870-875

9. Shivalkar B, Dhondt D, Goovaerts I et al (2006) Flow mediated dilatation and cardiac function in type 1 diabetes mellitus. Am J Cardiol 97:77-82

10. Fang ZY, Schull-Meade R, Leano R, Mottram PM, Prins JB, Marwick TH (2005) Screening for heart disease in diabetic subjects. Am Heart J 149:349-354

11. Ernande L, Bergerot C, Rietzschel ER et al (2011) Diastolic dysfunction in patients with type 2 diabetes mellitus: is it really the first marker of diabetic cardiomyopathy? J Am Soc Echocardiogr 24: $1268-1275$

12. Bugger H, Abel ED (2009) Rodent models of diabetic cardiomyopathy. Dis Model Mech 2:454-466

13. Wu KK, Huan Y (2007) Diabetic atherosclerosis mouse models. Atherosclerosis 191:241-249

14. Goldin A, Beckman JA, Schmidt AM, Creager MA (2006) Advanced glycation end products: sparking the development of diabetic vascular injury. Circulation 114:597-605

15. Norton GR, Candy G, Woodiwiss AJ (1996) Aminoguanidine prevents the decreased myocardial compliance produced by streptozotocin-induced diabetes mellitus in rats. Circulation 93: 1905-1912 
16. Aragno M, Mastrocola R, Medana C et al (2006) Oxidative stressdependent impairment of cardiac-specific transcription factors in experimental diabetes. Endocrinology 147:5967-5974

17. Bidasee KR, Zhang Y, Shao CH et al (2004) Diabetes increases formation of advanced glycation end products on Sarco(endo)plasmic reticulum Ca2+ -ATPase. Diabetes 53: 463-473

18. Kranstuber AL, del Rio C, Biesiadecki BJ et al (2012) Advanced glycation end product cross-link breaker attenuates diabetesinduced cardiac dysfunction by improving sarcoplasmic reticulum calcium handling. Front Physiol 3:292

19. Ma H, Li SY, Xu P et al (2009) Advanced glycation endproduct (AGE) accumulation and AGE receptor (RAGE) up-regulation contribute to the onset of diabetic cardiomyopathy. J Cell Mol Med 13:1751-1764

20. Regan TJ, Lyons MM, Ahmed SS et al (1977) Evidence for cardiomyopathy in familial diabetes mellitus. J Clin Invest 60:884-899

21. Shimizu M, Umeda K, Sugihara N et al (1993) Collagen remodelling in myocardia of patients with diabetes. J Clin Pathol 46:32-36

22. Westermann D, Rutschow S, Jager S et al (2007) Contributions of inflammation and cardiac matrix metalloproteinase activity to cardiac failure in diabetic cardiomyopathy: the role of angiotensin type 1 receptor antagonism. Diabetes 56:641-646

23. Singh VP, Le B, Khode R, Baker KM, Kumar R (2008) Intracellular angiotensin II production in diabetic rats is correlated with cardiomyocyte apoptosis, oxidative stress, and cardiac fibrosis. Diabetes 57:3297-3306

24. Mizushige K, Yao L, Noma T et al (2000) Alteration in left ventricular diastolic filling and accumulation of myocardial collagen at insulin-resistant prediabetic stage of a type II diabetic rat model. Circulation 101:899-907

25. Chiu J, Farhangkhoee H, Xu BY, Chen S, George B, Chakrabarti S (2008) PARP mediates structural alterations in diabetic cardiomyopathy. J Mol Cell Cardiol 45:385-393

26. Van Linthout S, Seeland U, Riad A et al (2008) Reduced MMP-2 activity contributes to cardiac fibrosis in experimental diabetic cardiomyopathy. Basic Res Cardiol 103:319-327

27. Diamant M, Lamb HJ, Smit JW, de Roos A, Heine RJ (2005) Diabetic cardiomyopathy in uncomplicated type 2 diabetes is associated with the metabolic syndrome and systemic inflammation. Diabetologia 48:1669-1670

28. Tschope C, Walther T, Escher F et al (2005) Transgenic activation of the kallikrein-kinin system inhibits intramyocardial inflammation, endothelial dysfunction and oxidative stress in experimental diabetic cardiomyopathy. FASEB J 19:2057-2059

29. Westermann D, Rutschow $S$, van Linthout $S$ et al (2006) Inhibition of p38 mitogen-activated protein kinase attenuates left ventricular dysfunction by mediating pro-inflammatory cardiac cytokine levels in a mouse model of diabetes mellitus. Diabetologia 49:2507-2513

30. Westermann $D$, van Linthout $S$, Dhayat $S$ et al (2007) Cardioprotective and anti-inflammatory effects of interleukin converting enzyme inhibition in experimental diabetic cardiomyopathy. Diabetes 56:1834-1841

31. Rajesh M, Batkai S, Kechrid M et al (2012) Cannabinoid 1 receptor promotes cardiac dysfunction, oxidative stress, inflammation, and fibrosis in diabetic cardiomyopathy. Diabetes 61:716-727

32. Westermann D, van Linthout S, Dhayat S et al (2007) Tumor necrosis factor-alpha antagonism protects from myocardial inflammation and fibrosis in experimental diabetic cardiomyopathy. Basic Res Cardiol 102:500-507

33. Westermann D, Walther T, Savvatis K et al (2009) Gene deletion of the kinin receptor B1 attenuates cardiac inflammation and fibrosis during the development of experimental diabetic cardiomyopathy. Diabetes 58:1373-1381

34. Wang ZV, Rothermel BA, Hill JA (2010) Autophagy in hypertensive heart disease. J Biol Chem 285:8509-8514
35. Rajesh M, Mukhopadhyay P, Batkai S et al (2009) Xanthine oxidase inhibitor allopurinol attenuates the development of diabetic cardiomyopathy. J Cell Mol Med 13:2330-2341

36. Li J, Zhu H, Shen E, Wan L, Arnold JM, Peng T (2010) Deficiency of rac1 blocks NADPH oxidase activation, inhibits endoplasmic reticulum stress, and reduces myocardial remodeling in a mouse model of type 1 diabetes. Diabetes 59:2033-2042

37. Jadhav A, Tiwari S, Lee P, Ndisang JF (2013) The heme oxygenase system selectively enhances the anti-inflammatory macrophage-M2 phenotype, reduces pericardial adiposity, and ameliorated cardiac injury in diabetic cardiomyopathy in Zucker diabetic fatty rats. J Pharmacol Exp Ther 345:239-249

38. Ti Y, Xie GL, Wang ZH et al (2011) TRB3 gene silencing alleviates diabetic cardiomyopathy in a type 2 diabetic rat model. Diabetes 60: 2963-2974

39. Monji A, Mitsui T, Bando YK, Aoyama M, Shigeta T, Murohara T (2013) Glucagon-like peptide-1 receptor activation reverses cardiac remodeling via normalizing cardiac steatosis and oxidative stress in type 2 diabetes. Am J Physiol 305:H295-H304

40. Frustaci A, Kajstura J, Chimenti C et al (2000) Myocardial cell death in human diabetes. Circ Res 87:1123-1132

41. Cai L, Li W, Wang G, Guo L, Jiang Y, Kang YJ (2002) Hyperglycemia-induced apoptosis in mouse myocardium: mitochondrial cytochrome C-mediated caspase-3 activation pathway. Diabetes 51:1938-1948

42. Huynh K, Kiriazis H, Du XJ et al (2013) Targeting the upregulation of reactive oxygen species subsequent to hyperglycemia prevents type 1 diabetic cardiomyopathy in mice. Free Radic Biol Med 60: 307-317

43. Varma A, Das A, Hoke NN, Durrant DE, Salloum FN, Kukreja RC (2012) Anti-inflammatory and cardioprotective effects of tadalafil in diabetic mice. PLoS One 7:e45243

44. Chowdhry MF, Vohra HA, Galinanes M (2007) Diabetes increases apoptosis and necrosis in both ischemic and nonischemic human myocardium: role of caspases and poly-adenosine diphosphateribose polymerase. J Thorac Cardiovasc Surg 134:124-131

45. Sari FR, Watanabe K, Thandavarayan RA et al (2010) 14-3-3 protein protects against cardiac endoplasmic reticulum stress (ERS) and ERS-initiated apoptosis in experimental diabetes. J Pharmacol Sci 113:325-334

46. Ares-Carrasco S, Picatoste B, Benito-Martin A et al (2009) Myocardial fibrosis and apoptosis, but not inflammation, are present in long-term experimental diabetes. Am J Physiol Heart Circ Physiol 297:H2109-H2119

47. Bojunga J, Nowak D, Mitrou PS, Hoelzer D, Zeuzem S, Chow KU (2004) Antioxidative treatment prevents activation of deathreceptor- and mitochondrion-dependent apoptosis in the hearts of diabetic rats. Diabetologia 47:2072-2080

48. Kajstura J, Fiordaliso F, Andreoli AM et al (2001) IGF-1 overexpression inhibits the development of diabetic cardiomyopathy and angiotensin II-mediated oxidative stress. Diabetes 50:1414-1424

49. Andrabi SA, Dawson TM, Dawson VL (2008) Mitochondrial and nuclear cross talk in cell death: parthanatos. Ann N Y Acad Sci 1147:233-241

50. Puthanveetil P, Zhang D, Wang Y et al (2012) Diabetes triggers a PARP1 mediated death pathway in the heart through participation of FoxO1. J Mol Cell Cardiol 53:677-686

51. Orea-Tejeda A, Colin-Ramirez E, Castillo-Martinez L et al (2007) Aldosterone receptor antagonists induce favorable cardiac remodeling in diastolic heart failure patients. Rev Invest Clin 59:103-107

52. Brown L, Wall D, Marchant C, Sernia C (1997) Tissue-specific changes in angiotensin II receptors in streptozotocin-diabetic rats. J Endocrinol 154:355-362

53. Li SY, Yang X, Ceylan-Isik AF, Du M, Sreejayan N, Ren J (2006) Cardiac contractile dysfunction in Lep/Lep obesity is accompanied 
by NADPH oxidase activation, oxidative modification of sarco(endo)plasmic reticulum Ca2+-ATPase and myosin heavy chain isozyme switch. Diabetologia 49:1434-1446

54. Fauconnier J, Lanner JT, Zhang SJ et al (2005) Insulin and inositol 1,4,5-trisphosphate trigger abnormal cytosolic $\mathrm{Ca} 2+$ transients and reveal mitochondrial $\mathrm{Ca} 2+$ handling defects in cardiomyocytes of ob/ob mice. Diabetes 54:2375-2381

55. Van den Bergh A, Vanderper A, Vangheluwe P et al (2008) Dyslipidaemia in type II diabetic mice does not aggravate contractile impairment but increases ventricular stiffness. Cardiovasc Res 77:371-379

56. Belke DD, Swanson EA, Dillmann WH (2004) Decreased sarcoplasmic reticulum activity and contractility in diabetic $\mathrm{db} / \mathrm{db}$ mouse heart. Diabetes 53:3201-3208

57. Pereira L, Matthes J, Schuster I et al (2006) Mechanisms of [Ca2+]i transient decrease in cardiomyopathy of $\mathrm{db} / \mathrm{db}$ type 2 diabetic mice. Diabetes 55:608-615

58. Ye G, Metreveli NS, Ren J, Epstein PN (2003) Metallothionein prevents diabetes-induced deficits in cardiomyocytes by inhibiting reactive oxygen species production. Diabetes 52:777-783

59. Ye G, Metreveli NS, Donthi RV et al (2004) Catalase protects cardiomyocyte function in models of type 1 and type 2 diabetes. Diabetes 53:1336-1343

60. Kralik PM, Ye G, Metreveli NS, Shem X, Epstein PN (2005) Cardiomyocyte dysfunction in models of type 1 and type 2 diabetes. Cardiovasc Toxicol 5:285-292

61. Lopaschuk GD, Tahiliani AG, Vadlamudi RV, Katz S, McNeill JH (1983) Cardiac sarcoplasmic reticulum function in insulin- or carnitine-treated diabetic rats. Am J Physiol Heart Circ Physiol 245:H969-H976

62. Flarsheim CE, Grupp IL, Matlib MA (1996) Mitochondrial dysfunction accompanies diastolic dysfunction in diabetic rat heart. Am J Physiol Heart Circ Physiol 271:H192-H202

63. Bugger H, Abel ED (2008) Molecular mechanisms for myocardial mitochondrial dysfunction in the metabolic syndrome. Clin Sci (Lond) 114:195-210

64. Pereira RO, Wende AR, Olsen C et al (2013) Inducible overexpression of GLUT1 prevents mitochondrial dysfunction and attenuates structural remodeling in pressure overload but does not prevent left ventricular dysfunction. J Am Heart Assoc 2:e000301

65. Luo M, Guan X, Luczak ED et al (2013) Diabetes increases mortality after myocardial infarction by oxidizing CaMKII. J Clin Invest 123:1262-1274

66. Erickson JR, Pereira L, Wang L et al (2013) Diabetic hyperglycaemia activates CaMKII and arrhythmias by O-linked glycosylation. Nature 502:372-376

67. Bugger H, Boudina S, Hu XX et al (2008) Type 1 diabetic akita mouse hearts are insulin sensitive but manifest structurally abnormal mitochondria that remain coupled despite increased uncoupling protein 3. Diabetes 57:2924-2932

68. Buchanan J, Mazumder PK, Hu P et al (2005) Reduced cardiac efficiency and altered substrate metabolism precedes the onset of hyperglycemia and contractile dysfunction in two mouse models of insulin resistance and obesity. Endocrinology 146:5341-5349

69. Peterson LR, Herrero P, Schechtman KB et al (2004) Effect of obesity and insulin resistance on myocardial substrate metabolism and efficiency in young women. Circulation 109:2191-2196

70. Finck BN, Lehman JJ, Leone TC et al (2002) The cardiac phenotype induced by PPARalpha overexpression mimics that caused by diabetes mellitus. J Clin Invest 109:121-130

71. Gibbs EM, Stock JL, McCoid SC et al (1995) Glycemic improvement in diabetic $\mathrm{db} / \mathrm{db}$ mice by overexpression of the human insulin-regulatable glucose transporter (GLUT4). J Clin Invest 95: $1512-1518$

72. Belke DD, Larsen TS, Gibbs EM, Severson DL (2000) Altered metabolism causes cardiac dysfunction in perfused hearts from diabetic $(\mathrm{db} / \mathrm{db})$ mice. Am J Physiol Endocrinol Metab 279: E1104-E1113

73. Wright JJ, Kim J, Buchanan J et al (2009) Mechanisms for increased myocardial fatty acid utilization following short-term high-fat feeding. Cardiovasc Res 82:351-360

74. Mazumder PK, O’Neill BT, Roberts MW et al (2004) Impaired cardiac efficiency and increased fatty acid oxidation in insulinresistant ob/ob mouse hearts. Diabetes 53:2366-2374

75. Boudina S, Sena S, Theobald H et al (2007) Mitochondrial energetics in the heart in obesity-related diabetes: direct evidence for increased uncoupled respiration and activation of uncoupling proteins. Diabetes 56:2457-2466

76. Boudina S, Sena S, O'Neill BT, Tathireddy P, Young ME, Abel ED (2005) Reduced mitochondrial oxidative capacity and increased mitochondrial uncoupling impair myocardial energetics in obesity. Circulation 112:2686-2695

77. Echtay KS, Roussel D, St-Pierre J et al (2002) Superoxide activates mitochondrial uncoupling proteins. Nature 415:96-99

78. Yagyu H, Chen G, Yokoyama M et al (2003) Lipoprotein lipase $(\mathrm{LpL})$ on the surface of cardiomyocytes increases lipid uptake and produces a cardiomyopathy. J Clin Invest 111:419-426

79. Chiu HC, Kovacs A, Ford DA et al (2001) A novel mouse model of lipotoxic cardiomyopathy. J Clin Invest 107:813-822

80. Chiu HC, Kovacs A, Blanton RM et al (2005) Transgenic expression of fatty acid transport protein 1 in the heart causes lipotoxic cardiomyopathy. Circ Res 96:225-233

81. Rijzewijk LJ, van der Meer RW, Smit JW et al (2008) Myocardial steatosis is an independent predictor of diastolic dysfunction in type 2 diabetes mellitus. J Am Coll Cardiol 52:1793-1799

82. Son NH, Yu S, Tuinei J et al (2010) PPAR $\gamma$-induced cardiolipotoxicity in mice is ameliorated by PPAR $\alpha$ deficiency despite increases in fatty acid oxidation. J Clin Invest 120:3443-3454

83. Zhou YT, Grayburn P, Karim A et al (2000) Lipotoxic heart disease in obese rats: implications for human obesity. Proc Natl Acad Sci U S A 97:1784-1789

84. Lee Y, Naseem RH, Park BH et al (2006) Alpha-lipoic acid prevents lipotoxic cardiomyopathy in acyl CoA-synthase transgenic mice. Biochem Biophys Res Commun 344:446-452

85. Lee Y, Naseem RH, Duplomb L et al (2004) Hyperleptinemia prevents lipotoxic cardiomyopathy in acyl CoA synthase transgenic mice. Proc Natl Acad Sci U S A 101:13624-13629

86. Listenberger LL, Ory DS, Schaffer JE (2001) Palmitate-induced apoptosis can occur through a ceramide-independent pathway. J Biol Chem 276:14890-14895

87. Ostrander DB, Sparagna GC, Amoscato AA, McMillin JB, Dowhan W (2001) Decreased cardiolipin synthesis corresponds with cytochrome c release in palmitate-induced cardiomyocyte apoptosis. J Biol Chem 276:38061-38067

88. Borradaile NM, Han X, Harp JD, Gale SE, Ory DS, Schaffer JE (2006) Disruption of endoplasmic reticulum structure and integrity in lipotoxic cell death. J Lipid Res 47:2726-2737

89. Brookheart RT, Michel CI, Listenberger LL, Ory DS, Schaffer JE (2009) The non-coding RNA gadd7 is a regulator of lipid-induced oxidative and endoplasmic reticulum stress. J Biol Chem 284:74467454

90. Michel CI, Holley CL, Scruggs BS et al (2011) Small nucleolar RNAs U32a, U33, and U35a are critical mediators of metabolic stress. Cell Metab 14:33-44

91. Anderson EJ, Kypson AP, Rodriguez E, Anderson CA, Lehr EJ, Neufer PD (2009) Substrate-specific derangements in mitochondrial metabolism and redox balance in the atrium of the type 2 diabetic human heart. J Am Coll Cardiol 54:1891-1898

92. Anderson EJ, Rodriguez E, Anderson CA, Thayne K, Chitwood WR, Kypson AP (2011) Increased propensity for cell death in diabetic human heart is mediated by mitochondrial-dependent pathways. Am J Physiol Heart Circ Physiol 300:H118-H124 
93. Kuo TH, Giacomelli F, Wiener J (1985) Oxidative metabolism of Polytron versus Nagarse mitochondria in hearts of genetically diabetic mice. Biochim Biophys Acta 806:9-15

94. Konig A, Bode C, Bugger H (2012) Diabetes mellitus and myocardial mitochondrial dysfunction: bench to bedside. Heart Fail Clin 8: 551-561

95. Cook SA, Varela-Carver A, Mongillo M et al (2010) Abnormal myocardial insulin signalling in type 2 diabetes and left-ventricular dysfunction. Eur Heart J 31:100-111

96. Shimizu I, Minamino T, Toko H et al (2010) Excessive cardiac insulin signaling exacerbates systolic dysfunction induced by pressure overload in rodents. J Clin Invest 120: $1506-1514$

97. Fullmer TM, Pei S, Zhu Y et al (2013) Insulin suppresses ischemic preconditioning-mediated cardioprotection through Akt-dependent mechanisms. J Mol Cell Cardiol 64:20-29

98. Battiprolu PK, Hojayev B, Jiang N et al (2012) Metabolic stressinduced activation of FoxO1 triggers diabetic cardiomyopathy in mice. J Clin Invest 122:1109-1118

99. Boudina S, Bugger H, Sena S et al (2009) Contribution of impaired myocardial insulin signaling to mitochondrial dysfunction and oxidative stress in the heart. Circulation 119:1272-1283

100. Bugger H, Riehle C, Jaishy B et al (2012) Genetic loss of insulin receptors worsens cardiac efficiency in diabetes. J Mol Cell Cardiol 52:1019-1026

101. Wallace DC (1992) Mitochondrial genetics: a paradigm for aging and degenerative diseases? Science 256:628-632

102. Lashin OM, Szweda PA, Szweda LI, Romani AM (2006) Decreased complex II respiration and HNE-modified SDH subunit in diabetic heart. Free Radic Biol Med 40:886-896

103. Turko IV, Li L, Aulak KS, Stuehr DJ, Chang JY, Murad F (2003) Protein tyrosine nitration in the mitochondria from diabetic mouse heart. Implications to dysfunctional mitochondria in diabetes. J Biol Chem 278:33972-33977

104. Shen X, Zheng S, Metreveli NS, Epstein PN (2006) Protection of cardiac mitochondria by overexpression of MnSOD reduces diabetic cardiomyopathy. Diabetes 55:798-805

105. Herlein JA, Fink BD, O’Malley Y, Sivitz WI (2009) Superoxide and respiratory coupling in mitochondria of insulin-deficient diabetic rats. Endocrinology 150:46-55

106. Serpillon S, Floyd BC, Gupte RS et al (2009) Superoxide production by NAD(P)H oxidase and mitochondria is increased in genetically obese and hyperglycemic rat heart and aorta before the development of cardiac dysfunction. The role of glucose-6-phosphate dehydrogenase-derived NADPH. Am J Physiol Heart Circ Physiol 297:H153-H162

107. Wold LE, Ceylan-Isik AF, Fang CX et al (2006) Metallothionein alleviates cardiac dysfunction in streptozotocin-induced diabetes: role of $\mathrm{Ca} 2+$ cycling proteins, NADPH oxidase, poly(ADP-Ribose) polymerase and myosin heavy chain isozyme. Free Radic Biol Med 40: 1419-1429

108. Levine B, Klionsky DJ (2004) Development by self-digestion: molecular mechanisms and biological functions of autophagy. Dev Cell 6:463-477

109. Nakai A, Yamaguchi O, Takeda T et al (2007) The role of autophagy in cardiomyocytes in the basal state and in response to hemodynamic stress. Nat Med 13:619-624

110. Yan L, Vatner DE, Kim SJ et al (2005) Autophagy in chronically ischemic myocardium. Proc Natl Acad Sci U S A 102:13807-13812

111. Zhu H, Tannous P, Johnstone JL et al (2007) Cardiac autophagy is a maladaptive response to hemodynamic stress. J Clin Invest 117: 1782-1793

112. Matsui Y, Takagi H, Qu X et al (2007) Distinct roles of autophagy in the heart during ischemia and reperfusion: roles of AMP-activated protein kinase and Beclin 1 in mediating autophagy. Circ Res 100: 914-922
113. Rothermel BA, Hill JA (2008) Autophagy in load-induced heart disease. Circ Res 103:1363-1369

114. Riehle C, Wende AR, Sena S et al (2013) Insulin receptor substrate signaling suppresses neonatal autophagy in the heart. J Clin Invest 123:5319-5333

115. Troncoso R, Vicencio JM, Parra V et al (2012) Energy-preserving effects of IGF-1 antagonize starvation-induced cardiac autophagy. Cardiovasc Res 93:320-329

116. Mellor KM, Bell JR, Young MJ, Ritchie RH, Delbridge LM (2011) Myocardial autophagy activation and suppressed survival signaling is associated with insulin resistance in fructose-fed mice. J Mol Cell Cardiol 50:1035-1043

117. Zhao J, Brault JJ, Schild A et al (2007) FoxO3 coordinately activates protein degradation by the autophagic/lysosomal and proteasomal pathways in atrophying muscle cells. Cell Metab 6: $472-483$

118. Hausenloy DJ, Mocanu MM, Yellon DM (2004) Cross-talk between the survival kinases during early reperfusion: its contribution to ischemic preconditioning. Cardiovasc Res 63:305-312

119. Sala-Mercado JA, Wider J, Undyala VV et al (2010) Profound cardioprotection with chloramphenicol succinate in the swine model of myocardial ischemia-reperfusion injury. Circulation 122:S179 S184

120. Nemchenko A, Chiong M, Turer A, Lavandero S, Hill JA (2011) Autophagy as a therapeutic target in cardiovascular disease. J Mol Cell Cardiol 51:584-593

121. Mellor KM, Reichelt ME, Delbridge LM (2011) Autophagy anomalies in the diabetic myocardium. Autophagy 7:1263-1267

122. Xie Z, Lau K, Eby B et al (2011) Improvement of cardiac functions by chronic metformin treatment is associated with enhanced cardiac autophagy in diabetic OVE26 mice. Diabetes 60:1770-1778

123. Kornfeld JW, Baitzel C, Konner AC et al (2013) Obesity-induced overexpression of miR-802 impairs glucose metabolism through silencing of Hnflb. Nature 494:111-115

124. Jordan SD, Kruger M, Willmes DM et al (2011) Obesity-induced overexpression of miRNA-143 inhibits insulin-stimulated AKT activation and impairs glucose metabolism. Nat Cell Biol 13:434 446

125. Zhou B, Li C, Qi W et al (2012) Downregulation of miR-181a upregulates sirtuin-1 (SIRT1) and improves hepatic insulin sensitivity. Diabetologia 55:2032-2043

126. Trajkovski M, Hausser J, Soutschek J et al (2011) MicroRNAs 103 and 107 regulate insulin sensitivity. Nature 474:649-653

127. Chen JF, Murchison EP, Tang R et al (2008) Targeted deletion of Dicer in the heart leads to dilated cardiomyopathy and heart failure. Proc Natl Acad Sci U S A 105:2111-2116

128. Feng B, Chen S, George B, Feng Q, Chakrabarti S (2010) miR133a regulates cardiomyocyte hypertrophy in diabetes. Diabetes Metab Res Rev 26:40-49

129. Kartha RV, Subramanian S (2010) MicroRNAs in cardiovascular diseases: biology and potential clinical applications. J Cardiovasc Transl Res 3:256-270

130. Katare R, Caporali A, Zentilin L et al (2011) Intravenous gene therapy with PIM-1 via a cardiotropic viral vector halts the progression of diabetic cardiomyopathy through promotion of prosurvival signaling. Circ Res 108:1238-1251

131. Xiao J, Luo X, Lin H et al (2007) MicroRNA miR-133 represses HERG K+ channel expression contributing to QT prolongation in diabetic hearts. J Biol Chem 282:12363-12367

132. Duisters RF, Tijsen AJ, Schroen B et al (2009) miR-133 and miR-30 regulate connective tissue growth factor: implications for a role of microRNAs in myocardial matrix remodeling. Circ Res 104:170 178

133. Bentwich I, Avniel A, Karov Y et al (2005) Identification of hundreds of conserved and nonconserved human microRNAs. Nat Genet 37:766-770 
134. Friedman RC, Farh KK, Burge CB, Bartel DP (2009) Most mammalian mRNAs are conserved targets of microRNAs. Genome Res 19:92-105

135. Khan O, La Thangue NB (2012) HDAC inhibitors in cancer biology: emerging mechanisms and clinical applications. Immunol Cell Biol 90: 85-94

136. McKinsey TA (2011) Isoform-selective HDAC inhibitors: closing in on translational medicine for the heart. J Mol Cell Cardiol 51: 491-496

137. Chuang DM, Leng Y, Marinova Z, Kim HJ, Chiu CT (2009) Multiple roles of HDAC inhibition in neurodegenerative conditions. Trends Neurosci 32:591-601

138. Chang S, McKinsey TA, Zhang CL, Richardson JA, Hill JA, Olson EN (2004) Histone deacetylases 5 and 9 govern responsiveness of the heart to a subset of stress signals and play redundant roles in heart development. Mol Cell Biol 24:8467-8476

139. Hamamori Y, Schneider MD (2003) HATs off to Hop: recruitment of a class I histone deacetylase incriminates a novel transcriptional pathway that opposes cardiac hypertrophy. J Clin Invest 112:824 826

140. Gaikwad AB, Sayyed SG, Lichtnekert J, Tikoo K, Anders HJ (2010) Renal failure increases cardiac histone h3 acetylation, dimethylation, and phosphorylation and the induction of cardiomyopathy-related genes in type 2 diabetes. Am J Pathol 176:1079-1083

141. Monkemann H, de Vriese AS, Blom HJ et al (2002) Early molecular events in the development of the diabetic cardiomyopathy. Amino Acids 23:331-336

142. Kuan CJ, al-Douahji M, Shankland SJ (1998) The cyclin kinase inhibitor p21WAF1, CIP1 is increased in experimental diabetic nephropathy: potential role in glomerular hypertrophy. J Am Soc Nephrol 9:986-993

143. Kaneto H, Kajimoto Y, Fujitani Y et al (1999) Oxidative stress induces $\mathrm{p} 21$ expression in pancreatic islet cells: possible implication in beta-cell dysfunction. Diabetologia 42:1093-1097

144. Li Z, Zhang T, Dai H et al (2007) Involvement of endoplasmic reticulum stress in myocardial apoptosis of streptozocin-induced diabetic rats. J Clin Biochem Nutr 41:58-67

145. Xu J, Wang G, Wang Y et al (2009) Diabetes- and angiotensin IIinduced cardiac endoplasmic reticulum stress and cell death: metallothionein protection. J Cell Mol Med 13:1499-1512

146. Lakshmanan AP, Harima M, Suzuki K et al (2013) The hyperglycemia stimulated myocardial endoplasmic reticulum (ER) stress contributes to diabetic cardiomyopathy in the transgenic nonobese type 2 diabetic rats: a differential role of unfolded protein response (UPR) signaling proteins. Int J Biochem Cell Biol 45:438 447

147. Liu ZW, Zhu HT, Chen KL et al (2013) Protein kinase RNA- like endoplasmic reticulum kinase (PERK) signaling pathway plays a major role in reactive oxygen species (ROS)-mediated endoplasmic reticulum stress-induced apoptosis in diabetic cardiomyopathy. Cardiovasc Diabetol 12:158

148. Liu J, Liu Y, Chen L, Wang Y, Li J (2013) Glucagon-like peptide-1 analog liraglutide protects against diabetic cardiomyopathy by the inhibition of the endoplasmic reticulum stress pathway. J Diabetes Res 2013:630537

149. Younce CW, Burmeister MA, Ayala JE (2013) Exendin-4 attenuates high glucose-induced cardiomyocyte apoptosis via inhibition of endoplasmic reticulum stress and activation of SERCA2a. Am J Physiol Cell Physiol 304:C508-C518 